\title{
Nash Equilibrium Solution In Trapezoidal Fuzzy Environment
}

\author{
Sibasis Bandyopadhyay ${ }^{1}$, Prasun Kumar Nayak ${ }^{2}$,Madhumangal Pal ${ }^{3}$ \\ ${ }^{1}$ Gourangdi R.K.S Institution,Asansol-713304,India \\ ${ }^{2}$ Bankura Christian College, Bankura, 722 101, India, \\ ${ }^{3}$ Department of Applied Mathematics with oceanology and Computer Programming, Vidyasagar University \\ Midnapore-721102,India
}

\begin{abstract}
In this paper the bimatrix game with symmetric trapezoidal pay -off is considered.At first trapezoidal number ranking method for such games is defined and then we define Nash equilibrium solution for pure strategies and mixed strategies. The inequality constraints involving trapezoidal co-efficients are reduced in their satisfactory crisp equivalent form and a satisfactory solution of the problem is established.Numerical example is given to illustrate the methodology.
\end{abstract}

Keywords:- Bi-Matrix game,Symmetric trapezoidal number,Nash equilibrium.

\section{INTRODUCTION}

Game theory is a mathematical tool using which conflicting interest situations are handled. In recent times much attention has been drawn to interval valued game, Nayak and Pal [1, 2, 3], Narayanan [4], Nishizaki[5]. In practical situations the pay-offs are given with in certain ranges rather than as an exact number. These uncertain situations are overcome when we use interval numbers as pay-offs. An interval number is an extension of a real number and also subset of a real line $\mathfrak{R}$, Moore [6]. Zimmermann [7] shows that $\alpha$ cut of a fuzzy number is an interval number. The method of solution of a matrix game using interval numbers was already established, Nayak and Pal [3]. In Narayanan [4], probability and possibility approaches have been used to solve a $2 \times 2$ interval game but no certain distribution function has been used. Moreover, reduction of an $m \times n$ game to a $2 \times 2$ sub game is a basic problem in an interval game. In dominance method [1], if the convex combination of any two rows(columns)of a pay-off matrix is dominated by the third row(column) then it indicates that the third move of the row(column) of the player will be an optimal move but we are not certain as to which one of the first two moves will be an optimal one.This disadvantage is overcome through the graphical method [2]. However, this is not the method of solution but a state of art technique, where an $m \times 2$ or $2 \times n$ particular type interval game is reduced to $2 \times 2$ interval subgames. But the situation may arise where more than two decision makers are involved and that situation is handled by bi-matrix game. Fuzziness, in bi-matrix games, were studied by many authors $[8,9,10]$. Nayak and Pal[11] described bi-matrix games with interval pay-off and its Nash equilibrium strategy. In this paper we have described bi-matrix game with trapezoidal fuzzy pay off. We have described the arithmetic operation and inequality relation of trapezoidal fuzzy numbers. We defined Nash equilibrium solution of such games and tried to get the solution as a trapezoidal fuzzy number. The numerical example illustrates the theory.

\section{SYMMETRIC TRAPEZOIDAL NUMBER}

Here e will pay attention on some definitions and notations of symmetric trapezoidal fuzzy number. our aim is to define a fuzzy symmetric trapezoidal matrix in space of matrices. The triangular symmetric fuzzy number has already been defined.Here we define the symmetric trapezoidal fuzzy number over the real line $\mathrm{L}$ in some different manner as

$$
L=\left\{a ; x \in \mathfrak{R} \text { and } a=a_{0}+(3 x-1) \alpha, x \in[0,1]\right\}
$$

Here at

$$
\begin{aligned}
& x=0, a=a_{0}-\alpha \\
& x=\frac{1}{3}, a=a_{0}
\end{aligned}
$$




$$
\begin{aligned}
& x=\frac{2}{3}, a=a_{0}+\alpha=a_{1}(\text { say }) \\
& x=1, a=a_{0}+2 \alpha=a_{1}+\alpha
\end{aligned}
$$

Where $a_{0}$ is the $1^{\text {st }}$ arithmetic mean value $\left(\right.$ for $\left.x=\frac{1}{3}\right), a_{1}$ is the $2^{\text {nd }}$ arithmetic mean value $\left(\right.$ for $\left.x=\frac{2}{3}\right), \underline{a}$ is the lower bound $($ for $x=0)$ and $\bar{a}$ is the upper bound $($ for $x=1)$. The symmetric trapezoidal membership function is defined on $[0,1]$ as

$$
\prod(x)=\left\{\begin{array}{cc}
0 ; & \text { when } 0 \leq x, \\
3 x ; & \text { when } 0<x<\frac{1}{3} \\
1 ; & \text { when } \frac{1}{3} \leq x \leq \frac{2}{3} \\
3(1-x) ; & \text { when } \frac{2}{3}<x<1 \\
0 ; & \text { when } x \geq 1
\end{array}\right.
$$

Here one observation can be made that we use three parameters to represent a symmetric trapezoidal fuzzy number $\left\langle m, m^{\prime}, \omega\right\rangle$ though four parameters are required to represent a general trapezoidal fuzzy number.This representation has been made for the simplicity of the computation.

\section{TRAPEZOIDAL ARITHMETIC}

An extension of ordinary arithmetic to trapezoidal fuzzy number is known as trapezoidal arithmetic.

Let $\mathrm{A}=\left\langle a_{1}, a_{2}, \omega\right\rangle$ and $\mathrm{B}=\left\langle b_{1}, b_{2}, \omega^{\prime}\right\rangle$ be two trapezoidal numbers.The arithmetic and multiplication by a real number $\mathrm{C}$ are defined as follow:-

1. The sum of two trapezoidal numbers is a trapezoidal numberi.e

$$
\begin{gathered}
A+B=\left\langle a_{1}, a_{2}, \omega\right\rangle+\left\langle b_{1}, b_{2}, \omega^{\prime}\right\rangle \\
=\left\langle a_{1}+b_{1}, a_{2}+b_{2}, \omega+\omega^{\prime}\right\rangle
\end{gathered}
$$

2. The subtraction of two trapezoidal fuzzy number is also a trapezoidal fuzzy number i.e

$$
\begin{gathered}
A-B=\left\langle a_{1}, a_{2}, \omega\right\rangle-\left\langle b_{1}, b_{2}, \omega^{\prime}\right\rangle \\
=\left\langle a_{1}-b_{2}, a_{2}-b_{1}, \omega+\omega^{\prime}\right\rangle
\end{gathered}
$$

3. If $c \neq 0$ be a scalar then $c A=\left\langle c a_{1}, c a_{2}, c \omega\right\rangle$ if $c \geq 0$ and $c A=\left\langle c a_{2}, c a_{1},-c \omega\right\rangle$ if $c<0$.

4. The product of two trapezoidal number is given by

$$
\begin{aligned}
& A B=\left\langle\operatorname { m i n } \left[\left\{a_{1} b_{1}-\frac{1}{2}\left(\omega^{\prime} a_{1}+\omega b_{1}\right)+\frac{1}{4} \omega \omega^{\prime}\right\},\left\{a_{1} b_{2}+\frac{1}{2}\left(\omega^{\prime} a_{1}-\omega b_{2}\right)-\frac{1}{4} \omega^{\prime} \omega\right\},\left\{a_{2} b_{1}+\frac{1}{2}\left(\omega b_{1}-\omega^{\prime} a_{2}\right)-\frac{1}{4} \omega \omega^{\prime}\right\},\right.\right. \\
& \left\{a_{2} b_{2}+\frac{1}{2}\left(\omega^{\prime} a_{2}+\omega b_{2}\right)+\frac{1}{4} \omega \omega^{\prime}\right], \max \left[a_{1} b_{1}-\frac{1}{2}\left(\omega^{\prime} a_{1}+\omega b_{1}\right)+\frac{1}{4} \omega \omega^{\prime}\right\},\left\{a_{1} b_{2}+\frac{1}{2}\left(\omega^{\prime} a_{1}-\omega b_{2}\right)-\frac{1}{4} \omega^{\prime} \omega\right\},
\end{aligned}
$$




$$
\left.\left.\left\{a_{2} b_{1}+\frac{1}{2}\left(\omega b_{1}-\omega^{\prime} a_{2}\right)-\frac{1}{4} \omega \omega^{\prime}\right\},\left\{a_{2} b_{2}+\frac{1}{2}\left(\omega^{\prime} a_{2}+\omega b_{2}\right)+\frac{1}{4} \omega \omega^{\prime}\right\}\right],(\max -\min )\right\rangle
$$

5. The division of these two interval numbers $\mathrm{A}$ and $\mathrm{B}(\neq 0)$ is

$$
\begin{aligned}
& \frac{A}{B}=\left\langle\min \left[\frac{2 a_{1}-\omega}{2 b_{1}-\omega^{\prime}}, \frac{2 a_{1}-\omega}{2 b_{2}+\omega^{\prime}}, \frac{2 a_{2}+\omega}{2 b_{1}-\omega^{\prime}}, \frac{2 a_{2}+\omega}{2 b_{2}+\omega^{\prime}}\right],\right. \\
& \left.\max \left[\frac{2 a_{1}-\omega}{2 b_{1}-\omega^{\prime}}, \frac{2 a_{1}-\omega}{2 b_{2}+\omega^{\prime}}, \frac{2 a_{2}+\omega}{2 b_{1}-\omega^{\prime}}, \frac{2 a_{2}+\omega}{2 b_{2}+\omega^{\prime}}\right],(\max -\min )\right\rangle
\end{aligned}
$$

provided $2 b_{1} \neq \omega^{\prime}, 2 b_{2}+\omega^{\prime} \neq 0$

6. $A-A \neq 0$

7. $\frac{A}{A} \neq 1$

Example: If $A=\langle 2,5,3\rangle$ and $B=\langle 3,9,6\rangle$ are two trapezoidal fuzzy numbers then $A+B=\langle 5,14,9\rangle$ ,A-B $=\langle-7,2,9\rangle, \mathrm{AB}=\langle 0,78,78\rangle, \frac{A}{B}=$ does not exist as $2 b_{1}=\omega^{\prime}$

\section{INEQUALITY RELATION WITH TRAPEZOIDAL NUMBERS}

A comparison between trapezoidal numbers is given in this section.

case:-1 Let $A=\left\langle a_{L}, a_{R}, \omega\right\rangle$ and $B=\left\langle b_{L}, b_{R}, \omega^{\prime}\right\rangle$ be two trapezoidal numbers in $\mathrm{T}(\mathfrak{R})$, the set of trapezoidal numbers.Here we make analogous order relation to Moore[6] as $A<B$ (A is strictly less than B)iff $\omega+\omega^{\prime}<2\left(b_{L}-a_{R}\right)$ Here this relation is an extension of ' $<$ ' on real line. This relation seems to be strict order relation that $\mathrm{A}$ is smaller than $\mathrm{B}$.

Example:- Let $A=\langle 3,4,1\rangle$ and $B=\langle 8,14,6\rangle$ be two trapezoidal numbers. Here $\omega+\omega^{\prime}=1+6=7$ and $2\left(b_{L}-a_{R}\right)=2(8-4)=2 \times 4=8 . \Rightarrow \omega+\omega^{\prime}<2\left(b_{L}-a_{R}\right)$.

$\Rightarrow A<B$.

case:-2Let

$$
A=\left\langle a_{L}, a_{R}, \omega\right\rangle \quad \text { and }
$$$$
B=\left\langle b_{L}, b_{R}, \omega^{\prime}\right\rangle \in T(\mathfrak{R})
$$

s.t $2 a_{L}-\omega \leq 2 b_{L}-\omega^{\prime}<2 b_{R}+\omega^{\prime} \leq 2 a_{R}+\omega$. Then $\mathrm{B}$ contained in $\mathrm{A}$, denoted by $B \subseteq A$ which is the extension of the concept of the set inclusion.The ranking order of $\mathrm{A}$ and $\mathrm{B}$ can be defined as

$$
A \vee B=\left\{\begin{array}{l}
A, \text { if the player is optimistic } \\
B, \text { if the player is pessimistic }
\end{array}\right.
$$

. Where the notation $A \vee B$ represents maximum among two trapezoidals and

$$
A \wedge B=\left\{\begin{array}{l}
B, \text { if the player is optimistic } \\
A, \text { if the player is pessimistic }
\end{array}\right.
$$

Where the notation $A \wedge B$ represents minimum among two trapezoidals.

Example:-Let $A\langle 3,9,6\rangle$ and $B=\langle 4,8,4\rangle$ are two trapezoidals s.t $B \subseteq A$.For optimistic player $A \bigvee B=A$ and $A \wedge B=B$. For pessimistic player $A \vee B=B$ and $A \wedge B=A$.

Case 3:- Let us consider the case where the trapezoidal A and $B$ are not contained in others their some portions are common.In that case we define acceptability index to compare and order two trapezoidal numbers.

\subsection{Acceptability Index}


We use Acceptability indexAI to compare and order any two trapezoidal numbers on the real line in terms of value as in $[12,2]$, which are used throughout the paper. Let us consider two symmetric trapezoidal $\left\langle m_{1}, m_{1}^{\prime}, \omega_{1}\right\rangle$ and $\left\langle m_{2}, m_{2}^{\prime}, \omega_{2}\right\rangle$.

Definition 1 For $m_{1}+m_{1}^{\prime} \leq m_{2}+m_{2}^{\prime}$ and $\omega_{1}+\omega_{2} \neq 0$, the value judgement index or acceptability index

(AI) of the premise $A^{\prime} \prec B^{\prime}$ is defined by

$$
A I\left(A^{\prime} \prec B^{\prime}\right)=\frac{\left(m_{2}+m_{2}^{\prime}\right)-\left(m_{1}+m_{1}^{\prime}\right)}{2\left(\omega_{1}+\omega_{2}\right)},
$$

which is the value judgement by which $A^{\prime}$ is inferior to $B^{\prime}$ ( $B^{\prime}$ is superior to $A^{\prime}$ ) in terms of value. Here 'inferior to', 'superior to', are analogous to 'less than', 'greater than', respectively.

The above observations can be put into a compact form as follows

$$
A^{\prime} \vee B^{\prime}= \begin{cases}B^{\prime}, & \text { if } A I\left(A^{\prime} \prec B^{\prime}\right)>0 \\ A^{\prime}, & \text { if } A I\left(A^{\prime} \prec B^{\prime}\right)=0 \text { and } \omega_{1}<\omega_{2} \text { and } D M \text { is pessimistic } \\ B^{\prime}, & \text { if } A I\left(A^{\prime} \prec B^{\prime}\right)=0 \text { and } \omega_{1}<\omega_{2} \text { and } D M \text { is optimistic. }\end{cases}
$$

Similarly, in the following we have given another function $\max ^{\prime}$ which determines the maximum between two trapezoidal numbers.

\section{Function $\max ^{\prime}\left(A^{\prime}, B^{\prime}\right)$}

if $A=B$ then maximum $=A^{\prime}$;

else

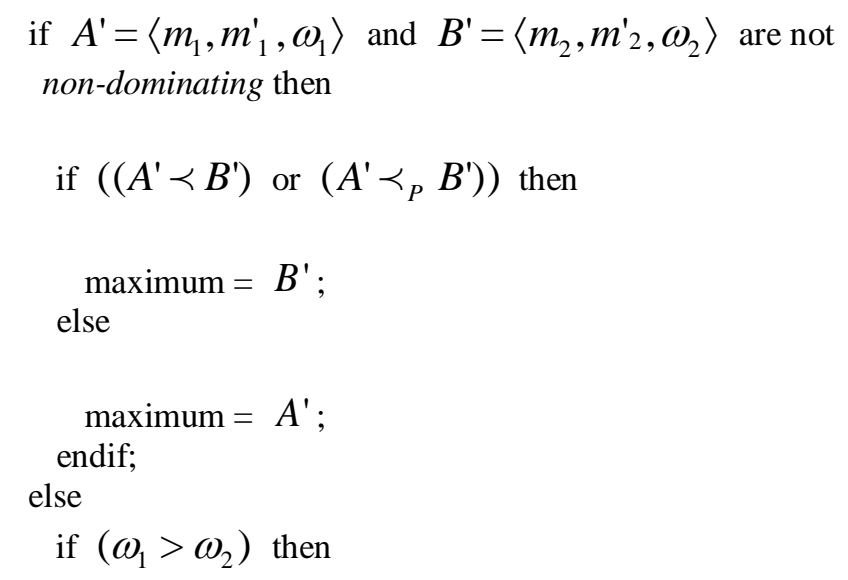

if the decision maker is optimistic maximum $=A^{\prime}$;

if the decision maker is pessimistic maximum $=B^{\prime}$; endif; 
endif;

endif;

return(maximum);

End Function.

Similarly, if $m_{1}+m_{1}^{\prime} \geq m_{2}+m_{2}^{\prime}$ and $\omega_{1} \geq \omega_{2}$, then there also exist a strict preference relation between $A^{\prime}$

and $B^{\prime}$. Thus similar observations can be put into a compact form as

$$
A^{\prime} \wedge B^{\prime}= \begin{cases}B^{\prime}, & \text { if } A I\left(B^{\prime} \prec A^{\prime}\right)>0 \\ A^{\prime}, & \text { if } A I\left(B^{\prime} \prec A^{\prime}\right)=0 \text { and } \omega_{1}>\omega_{2} \text { and } D M \text { is pessimistic } \\ B^{\prime}, & \text { if } A I\left(B^{\prime} \prec A^{\prime}\right)=0 \text { and } \omega_{1}>\omega_{2} \text { and DM is optimistic. }\end{cases}
$$

\section{INEQUALITY CONSTRAINTS INVOLVING TRAPEZOIDAL COEFFICIENTS}

In this section we concentrate on the LPP in which both the objective function and coefficients of constraints are all trapezoidal fuzzy numbers.Linear programming problems with interval coefficients have been studied by several researchers[3] by introducing some preference relations between interval numbers. We will follow analogous method to get inequality constraints involving trapezoidal coefficients. Following the definition of AI the inequality constraints involving trapezoidal coefficients are reduced in their crisp equivalent forms so that they can be solved easily. Let $A=\left\langle a_{L}, a_{R}, \omega\right\rangle$ and $B=\left\langle b_{L}, b_{R}, \omega^{\prime}\right\rangle \in T(\Re)$ and $\mathrm{x}$ is a singleton variable.Then acceptability condition of the inequality constraints $A x \leq B$ may be defined as

$$
A I(A x \prec B) \geq 0 \Rightarrow m(A x)+m^{\prime}(A x) \leq m(B)+m^{\prime}(B) \ldots \ldots .(1)
$$

Hence a crisp equivalent form of trapezoidal equality relation may be defined as

$$
A x \leq B
$$

$$
\Rightarrow\left\{\begin{array}{c}
\left(2 a_{R}+\omega\right) x \leq\left(2 b_{R}+\omega^{\prime}\right) \\
\frac{m(A x)+m^{\prime}(A x)-m(B)-m^{\prime}(B)}{2 \omega(A x)+2 \omega(B)} \leq \alpha \in[0,1] \cdots\left(1^{*}\right)
\end{array}\right.
$$

Where ' $\leq$ ' denotes trapezoidal number inequality and $\alpha$ denotes the minimal acceptance degree of constraints fixed by decision maker.Similarly the inequality constraints $A x \leq B$, the satisfactory crisp equivalent can be defined in the form

$$
\Rightarrow\left\{\begin{array}{c}
A x \geq B \quad(7) \\
\left(2 a_{L}-\omega\right) \geq\left(2 b_{L}-\omega^{\prime}\right) \\
\frac{m(B)+m^{\prime}(B)-m(A x)-m^{\prime}(A x)}{2 \omega(A x)+2 \omega(B)} \leq \alpha \in[0,1] \cdots\left(2^{*}\right)
\end{array}\right.
$$

Let $\left\langle c_{L j}, c_{R j}, \omega_{c j}\right\rangle,\left\langle a_{L i j}, a_{R i j}, \omega_{a i j}\right\rangle,\left\langle b_{L i}, b_{R i}, c_{R i}\right\rangle \in T(\Re)$. We consider following trapezoidal number linear programming problem(TNLP)as

$$
\begin{aligned}
& \max f(x)=\sum\left\langle c_{L j}, c_{R j}, \omega_{c j}\right\rangle x_{j}(8) \\
& \text { s.t } \sum\left\langle a_{L i j}, a_{R i j}, \omega_{a i j}\right\rangle x_{j} \leq\left\langle b_{L i}, b_{R j}, \omega_{b i}\right\rangle
\end{aligned}
$$

When max denotes fuzzy maximization,the crisp number $x_{j}$ are unknown, $f(x)$ is the objective function,2nd relation denotes $\mathrm{n}$ inequality constraints, $\mathrm{m}$ denotes the number of constraints and $\mathrm{n}$ denotes the number of variables. This type of model is called TNLP.However classical LPP technique can not be applied to get solution unless the above trapezoidal valued structure be reduced into standard LPP structure.In particular when 
the parameters are degenerated into crisp real numbers,TNLP turns into classical LPP as

$$
\begin{aligned}
& \max Z=m\left(f(x)=\frac{1}{2}\left\{\sum\left[C_{L j}+C_{R j}\right] x_{j}\right\}=\frac{1}{2}\left[v_{L}+v_{R}\right]\right. \\
& s . t \sum\left(2 a_{L i j}+\omega_{a i j}\right) x_{j} \geq 2 v_{L}, i=1,2, . . m \\
& \sum\left[(1-\alpha)\left(2 a_{L i j}+\omega_{a i j}\right)+(1+\alpha)\left(2 a_{R i j}+\omega_{a i j}\right)\right] x_{j} \geq(1+\alpha) v_{L}+(1-\alpha) v_{R} \\
& \sum x_{j}=1, x_{j} \geq 0, j=1,2, . . n, \alpha \in[0,1]
\end{aligned}
$$

The solution of this type crisp optimization problem satisfies all constraints exactly.In the analogous trapezoidal valued problem the degree of satisfaction of objective(s)and constraints is minimized.

\section{TRAPEZOIDAL VALUED BI-MATRIX GAME}

The TVBG is a finite non cooperative two person game.It can be considered as a natural extension of classical game to cover situations in which outcome of a decision process does not necessarily dictate the verdict that what one player gains and other has to lose. The table showing how payments should be made at the end of the game is called a pay off matrix. Let 1 and 2 denote two decision makers(DM) and let $M=\{1,2,3 \ldots m\}$ and $N=\{1,2,3 \ldots n\}$ be the sets of all pure strategies available for DM 1 and 2 respectively. $\left\langle\alpha_{i j}, \beta_{i j}, \omega_{i j}\right\rangle$, $\left\langle\gamma_{i j}, \delta_{i j}, \omega_{i j}^{\prime}\right\rangle$ denote pay offs that DM 1 and 2 recieve when DM 1 plays pure strategy i and player 2 plays pure strategy $\mathrm{j}$. Then we have following pay off matrices:-

$$
\begin{aligned}
& B_{1} \quad B_{2} \quad \cdots \quad B_{n} \\
& A=\begin{array}{c}
A_{1} \\
A_{2} \\
\vdots \\
A_{m}
\end{array}\left(\begin{array}{ccc}
\left\langle\alpha_{11}, \beta_{11}, \omega_{11}\right\rangle & \left\langle\alpha_{12}, \beta_{12}, \omega_{12}\right\rangle & \cdots\left\langle\alpha_{1 n}, \beta_{1 n}, \omega_{1 n}\right\rangle \\
\left\langle\alpha_{21}, \beta_{21}, \omega_{21}\right\rangle & \left\langle\alpha_{22}, \beta_{22}, \omega_{22}\right\rangle & \cdots\left\langle\alpha_{2 n}, \beta_{2 n}, \omega_{2 n}\right\rangle \\
\vdots & \vdots & \vdots \\
\left\langle\alpha_{m 1}, \beta_{m 1}, \omega_{m 1}\right\rangle & \left\langle\alpha_{m 2}, \beta_{m 2}, \omega_{m 2}\right\rangle & \cdots\left\langle\alpha_{m n}, \beta_{m n}, \omega_{m n}\right\rangle
\end{array}\right) \\
& B_{1} \quad B_{2} \quad \cdots \quad B_{n}
\end{aligned}
$$

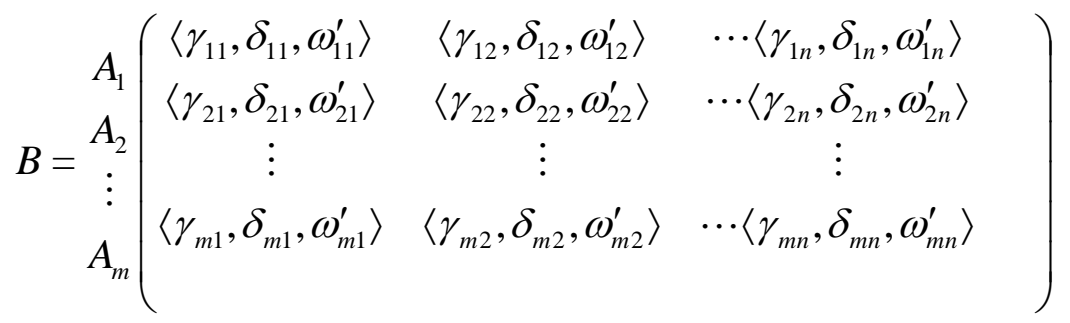

Where we assume that each of the two players chooses a strategy, a pay off for each of them and it is represented as trapezoidal number.We call this game a TVBG and denote the game by $\Gamma=\langle\{1,2\}, A, B\rangle$.

\section{NASH EQUILIBRIUM}

In this section we shall define concepts of Nash equilibrium strategies and investigate their properties.Bashar and olsder defined the concepts of Nash equilibrium solutions in bi-matrix games for single pair of pay off matrices and presented methodology for obtaining them.

\subsection{Pure Strategy}

Let 1,2 denote two decision makers(DM) and let $M=\{1,2,3, \ldots m\}$ and $N=\{1,2,3, \ldots n\}$ be the sets of 
all pure strategies available for DM1 and DM2 respectively. A Nash equilibrium solution represents an equilibrium point where each player reacts to other by choosing the option that gives him/her preference.

\subsection{Definition}

A pair of strategies \{row r,column $\mathrm{s}$ \} is said to constitute a Nash equilibrium solution for a TVBG $\Gamma$ if the following pair of inequalities are satisfied for all $i=1,2, \ldots m$ and $j=1,2, . . n$

$$
\bigwedge_{i}\left\langle\alpha_{i j}, \beta_{i j}, \omega_{i j}\right\rangle=\left\langle\alpha_{r s}, \beta_{r s}, \omega_{r s}\right\rangle \bigwedge_{j}\left\langle\gamma_{r j}, \delta_{r j}, \omega_{r s}^{\prime}\right\rangle=\left\langle\gamma_{i j}, \delta_{r s}, \omega_{r s}^{\prime}\right\rangle
$$

The pair $\left(\left\langle\alpha_{r s}, \beta_{r s}, \omega_{r s}\right\rangle,\left\langle\gamma_{r s}, \delta_{r s}, \omega_{r s}^{\prime}\right\rangle\right)$ is said to be a Nash equilibrium outcome of the TVBG.

\subsection{Mixed strategy}

A probabilistic situation arises when a player does not know the decision the other player takes but guesses what he can do and in that case the objective function is to maximize the expected gain. Such strategies are known as mixed strategies. Let $\mathfrak{R}_{+}^{m}$ and $\mathfrak{R}_{+}^{n}$ be the $m$ and $n$ dimensional nonnegative Euclidean spaces, respectively. Denote $x=\left(x_{1}, x_{2}, \cdots, x_{m}\right)^{T}$ and $y=\left(y_{1}, y_{2}, \ldots, y_{n}\right)^{T}$, respectively, where the symbol ' $T$ ' denotes the transpose of a vector. The strategy spaces for players $A$ and $B$ are denoted as

$$
\begin{aligned}
& S_{A}=\left\{\left(x_{1}, x_{2}, \cdots, x_{m}\right) \in \mathfrak{R}_{+}^{m}: x_{i} \in[0,1] ; i=1,2, \ldots, m \text { and } \sum_{i=1}^{m} x_{i}=1\right\} \\
& S_{B}=\left\{\left(y_{1}, y_{2}, \ldots, y_{n}\right) \in \mathfrak{R}_{+}^{n}: y_{i} \in[0,1] ; i=1,2, \ldots, n \text { and } \sum_{i=1}^{n} y_{i}=1\right\},
\end{aligned}
$$

respectively. Vectors $x \in S_{A}, y \in S_{B}$ are called mixed strategies of players $A$ and $B$, respectively.

Definition 2 (Trapezoidal expected pay-off ): If the mixed strategies $x=\left(x_{1}, x_{2}, \ldots, x_{m}\right)$ and $y=\left(y_{1}, y_{2}, \ldots, y_{n}\right)$ are proposed by players $A$ and $B$ respectively, then the expected pay-off of the player $A$ by player $B$ is defined by

$$
x^{T} A y=\sum_{j=1}^{n} \sum_{i=1}^{m} x_{i}\left\langle\alpha_{i j}, \beta_{i j}, \omega_{i j}\right\rangle y_{j} \text {.and } x^{T} B y=\sum_{j=1}^{n} \sum_{i=1}^{m} x_{i}\left\langle\gamma_{i j}, \delta_{i j}, \omega_{i j}^{\prime}\right\rangle y_{j} .
$$

Addition and other composition rules on trapezoidal numbers (discussed in section 2.2) are used in this definition (14) of expected pay-offs.

Definition 3 Nash equilibrium solution A pair $\left\{x^{*} \in S_{1}, y^{*}\right\}$ is called a Nash equilibrium solution to a TVBG $\Gamma$ in mixed strategies if the following inequalities are satisfied.

$$
x^{*^{T}} A y^{*} \leq x^{T} A y^{*} ; \forall x \in S_{I}, x^{* T} B y^{*} \leq x^{T} B y^{*} ; \forall y \in S_{I I}
$$

$x^{*}$ and $y^{*}$ are also called the optimal strategies for $\mathrm{A}$ and $\mathrm{B}$ respectively. Then the pair of intervals $V=\left\langle x^{* T} A y^{*}, x^{* T} B y^{*}\right\rangle$ is known as the Nash equilibrium outcome of the TVBG $\Gamma$ in mixed strategies, and the triplet $\left(x^{*}, y^{*}, V\right)$ is said to be a solution of TVBG.

\section{NUMERICAL EXAMPLE}

Consider a $2 \times 2$ TVBG with the pay-off matrices as 


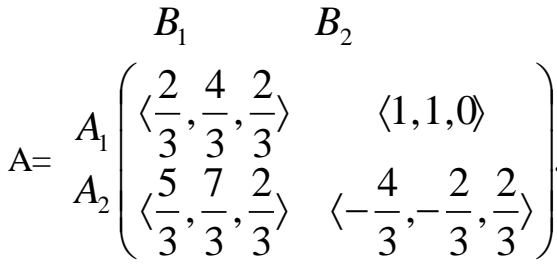

$$
\begin{aligned}
& B_{1} \quad B_{2}
\end{aligned}
$$

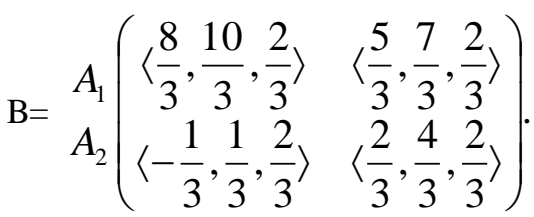

Here,Nash equilibrium solution in pure strategies does not exist w.r.t the ranking order described in section 4 . Here every $x \in S_{I}$ can be written as $\left(x_{1}, 1-x_{1}\right)^{T}$ with $0 \leq x_{1} \leq 1$ and similarly every $y \in S_{I I}$ as in $\left(y_{1}, 1-y_{1}\right)$ with $0 \leq y \leq 1$ we get the solution of the game as $\left\{x^{*}=\left(\frac{1}{2}, \frac{1}{2}\right)^{T}, y^{*}=\left(\frac{1}{2}, \frac{1}{2}\right)\right\}$ and $V=\left(\left\langle\frac{1}{6}, \frac{5}{6}, \frac{2}{3}\right\rangle,\left\langle\frac{7}{6}, \frac{11}{6}, \frac{2}{3}\right\rangle\right)$

\section{CONCLUSION}

In this paper,we have considered a bi-matrix game whose pay-off elements are symmetric trapezoidal fuzzy numbers. Arithmetic operations and inequality relations of the symmetric trapezoidal fuzzy numbers are described and Nash equilibrium strategies are explained.The numerical example establishes the theory on strong ground. It has wide industrial application where conflicting interest situations exist.

\section{REFERENCES}

[1]. P.K. Nayak and M. Pal, Solution of rectangular fuzzy games, OPSEARCH, 44(3),2009, 211-226.

[2]. P.K. Nayak and M. Pal, Solution of interval games using graphical method, Tamsui Oxford Journal of Mathematical Sciences, 22(1),2006,95-115.

[3]. P.K. Nayak and M. Pal, Linear programming technique to solve two person matrix games with interval pay-offs, Asia-Pacific Journal of Operational Research,26(2),2009,285-305.

[4]. A.L Narayan, A.R. Meenakshi and A.M.S. Ramasamy, Fuzzy Games, The Journal of Fuzzy Mathematics, 10,2002, 817-829.

[5]. I. Nishizaki and M. Sakawa, Equilibrium solutions for multiobjective bimatrix games incorporating fuzzy goals, Journal of Optimization Theory and Applications , 86 ,1995, 433-457.

[6]. R.E. Moore, Method and Application of Interval Analysis, SIAM, Philadelphia, 1979.

[7]. H.J. Zimmermann, Fuzzy mathematical programming, Computer Operational Research, 10(1983) 291-298.

[8]. C.R. Bector and S. Chandra, Fuzzy mathematical programming and fuzzy matrix game,Springer Verleg,New York(2000).

[9]. I.Nishijaki and M. Sakawa,Equilibrium solutions for multiobjective bi-matrix games incur-porating fuzzy goals,Journal of Optimization Theory and Applications, 86,1996,,433-457.

[10]. T. Maedea, Characterization of the equilibrium strategy of the bi-matrix game with fuzzy pay-off, Journal of Mathematical Analysis and Application, 251 (2000),885-896.

[11]. P.Nayak and M.Pal, The bi-matrix games with interval pay-offs and its Nash equilibrium strategy, The Journal of Fuzzy Mathematics, 17(2),2009,421-435.

[12]. A. Sengupta and T. K. Pal, On comparing interval numbers, European Journal of Operational Research, 127(1), 2000, 28 - 43. 